\title{
Modulation of Cardiac Performance by Motor Protein Gene Transfer
}

\author{
Todd J. Herron, ${ }^{a}$ Eric J. Devaney, ${ }^{b}$ AND Joseph M. Metzger ${ }^{a}$ \\ ${ }^{a}$ Department of Molecular and Integrative Physiology, \\ and \\ ${ }^{b}$ Department of Surgery, Division of Pediatric Cardiovascular Surgery, \\ University of Michigan Medical School, Ann Arbor, Michigan 48109, USA
}

\begin{abstract}
Gardiac muscle performance can be determined by factors intrinsic to each cardiac muscle cell, such as protein isoform expression. One protein whose expression plays a major role in determining cardiac performance is myosin. Myosin is the heart's molecular motor which transduces the chemical energy from ATP hydrolysis into the mechanical energy of each heartbeat. Alterations of myosin isoform expression are routinely associated with acquired and inherited cases of cardiomyopathy. For example, human heart failure is consistently associated with increased expression of a slow myosin motor isoform and a concomitant decreased expression of the heart's fast myosin motor isoform. Further, mutations of the cardiac myosin gene are the most common cause of inherited hypertrophic cardiomyopathy. Transgenic animal studies have provided insight into cardiac functional effects caused by myosin isoform gene switching (fast-to-slow myosin or slow-to-fast myosin) or by expression of a disease-related mutant motor. More direct structurefunction analysis using acute gene transfer of myosin motors provides evidence that the inotropic state of cardiac muscle can be affected by motor protein isoform shifting independent of intracellular calcium handling. Because most therapies for the diseased heart target intracellular calcium handling, acute gene transfer of cardiac molecular motors to modulate heart performance offers a novel therapeutic strategy for the compromised heart. Although the development of safe vectors for therapeutic myosin gene delivery are in their infancy, studies focused on acute genetic engineering of the heart's molecular motor will provide a foundation for therapeutic vector development and insight into mechanisms that contribute to cardiomyopathy.
\end{abstract}

Key words: myosin; molecular motor; acute genetic engineering; cardiac performance

\section{Introduction}

Oxygen and nutrient-rich blood is pumped to the body's tissues with each heartbeat. The force and pressure development of each heartbeat are due to the contraction and shortening of cardiac muscle. At the molecular level cardiac muscle contraction involves complex dynamic interactions between calcium and the myofilament proteins. Cardiac muscle force generation and shortening are initiated by calcium released from intracellular stores of the sarcoplasmic reticulum (SR) and executed by the activity of molecular motors within the myofilaments. A popular perspective is

Address for correspondence: Prof. Joseph M. Metzger, Ph.D., Department of Molecular and Integrative Physiology, University of Michigan, Ann Arbor, MI 48109. Voice: +734 647-6460; fax: +734 6476461 .

metzgerj@umich.edu that the level of force (or pressure) generation of cardiac muscle is determined primarily by the amount of calcium released from the SR and delivered to the myofilaments. However, mounting evidence suggests that cardiac muscle performance can also be altered independent of the calcium signal by the activity of the heart's molecular motor. In fact, a recent review article $^{1}$ has proposed a dominant role for molecular motors in the intrinsic regulation of cardiac performance.

Heart failure is a major health problem, affecting over 5 million people in the United States, and the numbers continue to grow. In cases of heart failure, cardiac performance is diminished and the pumping action of the heart is poor. As a result, the heart is unable to supply a sufficient amount of oxygenated and nutrient-rich blood to the tissues and organs that demand it. Common symptoms of heart failure include dyspnea, exercise intolerance, and edema. Current therapies manage the symptoms of heart failure 
and surgical intervention or cardiac transplantation is ultimately necessary. The development of novel therapies for heart failure is clearly necessary, and some investigators have predicted a shift in emphasis toward the development of gene-based therapy. ${ }^{2}$ One closely related pair of genes consistently associated with heart failure is the one that encodes the heart's molecular motors. $^{3-5}$

The importance of the heart's molecular motor, myosin, in the regulation of cardiac performance has been underscored in the past two decades. Since the first discovery that a myosin gene missense mutation can cause inherited hypertrophic cardiomyopathy $\left(\mathrm{HCM}^{6}\right.$ nearly 200 other disease-associated myosin mutations have been identified. Further, myosin missense mutations have also been implicated in other inherited cardiovascular diseases, including dilated cardiomyopathy (DCM), ${ }^{7,8}$ and in skeletal muscle myopathies. ${ }^{9}$ Since the cardiac phenotype of inherited cardiomyopathies can be similar to the phenotype of acquired heart failure, it is likely that alterations of myosin function contribute to the pathogenesis of more common forms of heart failure. The clinical importance of cardiac myosin makes it a good target for gene transfer experiments using viral vectors to study the molecular mechanisms that contribute to heart failure and for the development of novel gene-based therapies.

\section{Molecular Motors: Cardiac Myosin Isoforms}

Cardiac myosin belongs to the myosin II family of conventional myosins and is the molecular motor that drives myocardial contraction. Cardiac myosin is the most abundant protein within cardiac muscle and is the primary consumer of cellular energy, in the form of ATP. Muscular contraction is due primarily to cyclical interactions between interdigitating myosin and actin filaments. Myosin is an asymmetric molecule made up of two myosin heavy chains ( $\mathrm{MyHC}$ ) and two pairs of myosin light chains. The crystal structure of myosin has been solved ${ }^{10}$ and at its $\mathrm{N}$ terminus $\mathrm{MyHC}$ has a globular head domain which contains both an actinbinding site and an ATP binding and hydrolysis site (Fig. 1A). At its $\mathrm{C}$ terminus MyHC contains a rod domain made up of an $\alpha$-helical tail that interacts with the $\alpha$-helical tail of neighboring MyHC molecules to form the bipolar thick-filament backbone. Thus, in addition to hydrolyzing ATP as the heart's molecular motor, the $\mathrm{MyHC}$ also contributes to the structure of cardiac muscle. Two structurally and functionally distinct iso- forms of myosin are differentially expressed in cardiac muscle, namely, $\alpha$ - and $\beta$-MyHC isoforms (FIG. 1A). These isoforms can form hetero- and homodimers and these dimers have been separated electrophoretically and are referred to as myosin $\mathrm{V}_{1}(\alpha-\alpha$ homodimer), $\mathrm{V}_{2}(\alpha-\beta$ heterodimer $)$, and $\mathrm{V}_{3}(\beta-\beta$ homodimer $) .{ }^{11,12}$ The $\alpha$ - and $\beta$-myosin genes, MYH6 and MYH7, respectively, extend over $51 \mathrm{~kb}$ on chromosome 14 in humans ${ }^{13}$ and chromosome 11 in mouse separated by $4 \mathrm{~kb}$ of sequence between them. ${ }^{13}$ Transcription of each gene is independently controlled, but coordinately regulated, ${ }^{14}$ and changes throughout mammalian development. The $\mathrm{V}_{3}(\beta-\beta$ homodimer) myosin isoform is predominantly expressed during early development in the ventricles of all mammals and remains the predominant isoform throughout the lifetime of humans and larger mammals. The $\mathrm{V}_{1}(\alpha-\alpha$ homodimer $)$ isoform, on the other hand, predominates soon after birth in most small mammals and is the predominant isoform throughout the lifetime of mice and rats. ${ }^{15}$

Despite sharing $>90 \%$ amino acid homology, each molecular motor isoform functions in a distinct manner. For example, biochemical experiments have demonstrated that $\beta$-MyHC hydrolyzes ATP $\sim 3-7$ times slower than $\alpha-\mathrm{MyHC},{ }^{16,17}$ the "fast" motor in the heart. Using the in vitro motility assay, where actin propulsion by single myosin motors is measured, it has been demonstrated that $\alpha-\mathrm{MyHC}$ propels actin filaments $2-3$ times faster than $\beta-\mathrm{MyHC} .{ }^{17,18}$ These biochemical functional differences between the isoforms do not manifest as differences in force-generating capacity between the two isoforms. Using the laser trap assay, where the amount of isometric force produced by a single myosin molecule is measured, it has been demonstrated that $\alpha$ - and $\beta$-MyHC generate identical amounts of force, displace actin by the same extent, and only differ in their kinetics of ATP hydrolysis product release. ${ }^{19}$ Although differences in peak twitch tension are not apparent, the energetic economy and tension-time integral for preparations containing predominantly $\mathrm{V} 1$ myosin are less than that of preparations with predominantly V3 myosin. ${ }^{20,21}$ These kinetic differences in the myosin molecular motor isoforms manifest functionally as differences in the kinetics of force and power development in the context of single cardiac myocytes ${ }^{22}$ cardiac muscle strips, and whole heart function. ${ }^{23}$ FIGURE $1 \mathrm{~B}$, for example, demonstrates that the power output of single cardiac myocytes $^{24}$ is much lower in cardiac myocytes that exclusively express the $\beta-\mathrm{MyHC}$ isoform compared to cardiac myocytes that only express the faster $\alpha-\mathrm{MyHC}$ molecular motor isoform. Because maximum force production is not different between the 
A

$$
\alpha-\mathrm{MyHC}
$$

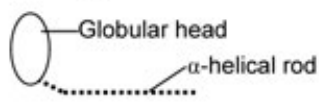

Faster ATPase activity (2-3times)

Faster molecular motor

\section{$\beta-\mathrm{MyHC}$}

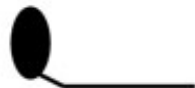

Slower ATPase activity

Slower molecular motor

B
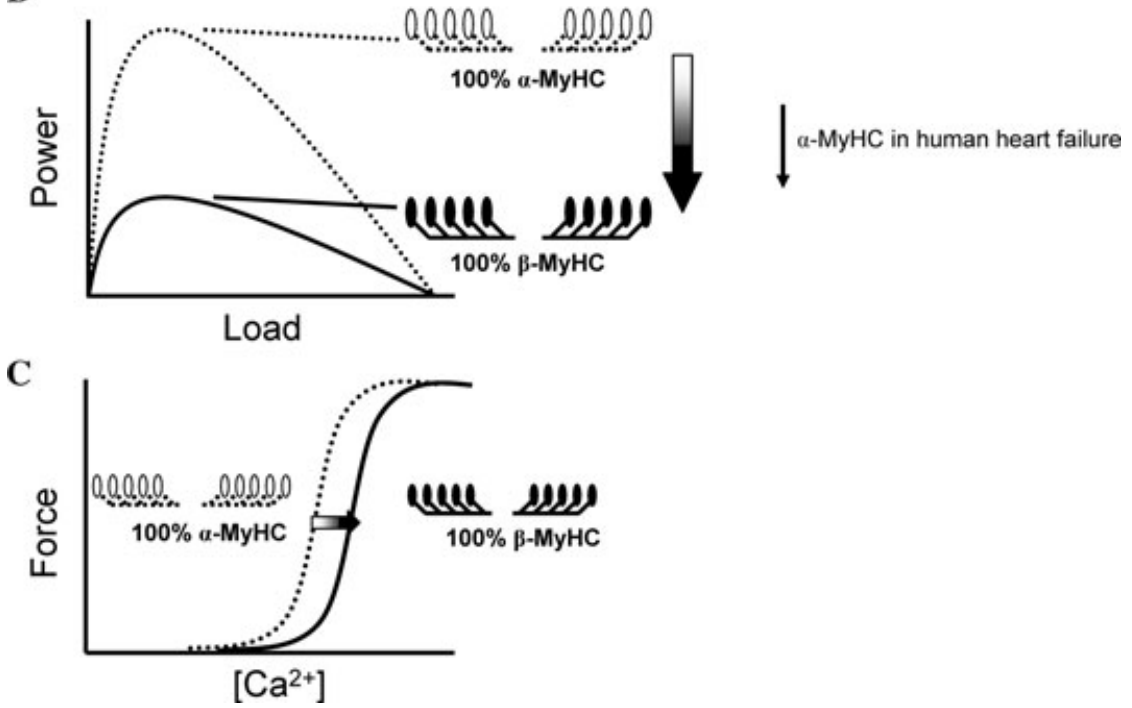

FIGURE 1. (A) Schematic representation of the cardiac MyHC molecule. Cardiac myosin is made up of a globular head domain and an $\alpha$-helical rod domain. Two distinct myosin isoforms are expressed in the heart: $\alpha$ - and $\beta-M y H C$. (B) Cardiac myosin isoforms determine the power output of single cardiac muscle cells (reproduced from Herron et al. ${ }^{24}$ with permission). (C) Myofilament calcium sensitivity is reduced in cardiac myocytes that express predominantly $\beta-\mathrm{MyHC}$.

isoforms, loaded shortening velocities (myocyte shortening against a clamped load as the heart does with each stroke) are faster in cardiac myocytes that express the faster $\alpha$-MyHC molecular motor. It has also been reported that cardiac myofilament responsiveness to calcium is depressed and the force-calcium relationship is shifted to the right in cardiac myocytes expressing the slower $\beta$-MyHC isoform ${ }^{25}$ (FIG. 1C). These effects of myosin isoform switching are also apparent at the level of the whole working heart ${ }^{23,26}$ (FIG. 2). Collectively, the literature supports a role for myosin isoforms as an important determinant of cardiac performance.

\section{Myosin Isoform Expression and Cardiovascular Disease}

Expression of the slow $\beta$-MyHC motor increases relative to $\alpha-\mathrm{MyHC}$ in rodent models of cardiovascular disease, including diabetes, ${ }^{27}$ hypothyroidism, ${ }^{22,25}$ cardiac hypertrophy, ${ }^{28}$ and in aging. ${ }^{29}$ In these instances the total amount of myosin remains unchanged, but the relative expression of each isoform changes. In rat models of cardiovascular disease, for example, the rat cardiac ventricle transforms and expression of the $\alpha-\mathrm{MyHC}$ isoform is replaced by expression of the slower $\beta$-MyHC motor. ${ }^{29,30}$ Since this switch of myosin isoform expression occurs in a plethora of models of heart disease, increased expression of the $\beta$-MyHC motor expression is generally accepted as a molecular biomarker of cardiac disease in rodents.

Alterations of cardiac MyHC isoform expression may play a role in the pathogenesis of human heart failure as well. The "normal" human cardiac ventricles express predominantly $\beta$-MyHC (85-90\% of total myosin) and a small amount of the faster $\alpha-\mathrm{MyHC}$ $\left(10-15 \%\right.$ of total myosin) motor. ${ }^{4}$ The failing human heart is consistently associated with a loss of $\alpha-\mathrm{MyHC}$ and exclusive expression of the slow $\beta$-MyHC motor. Patients undergoing successful treatment of heart failure with pharmacologic or surgical interventions have 


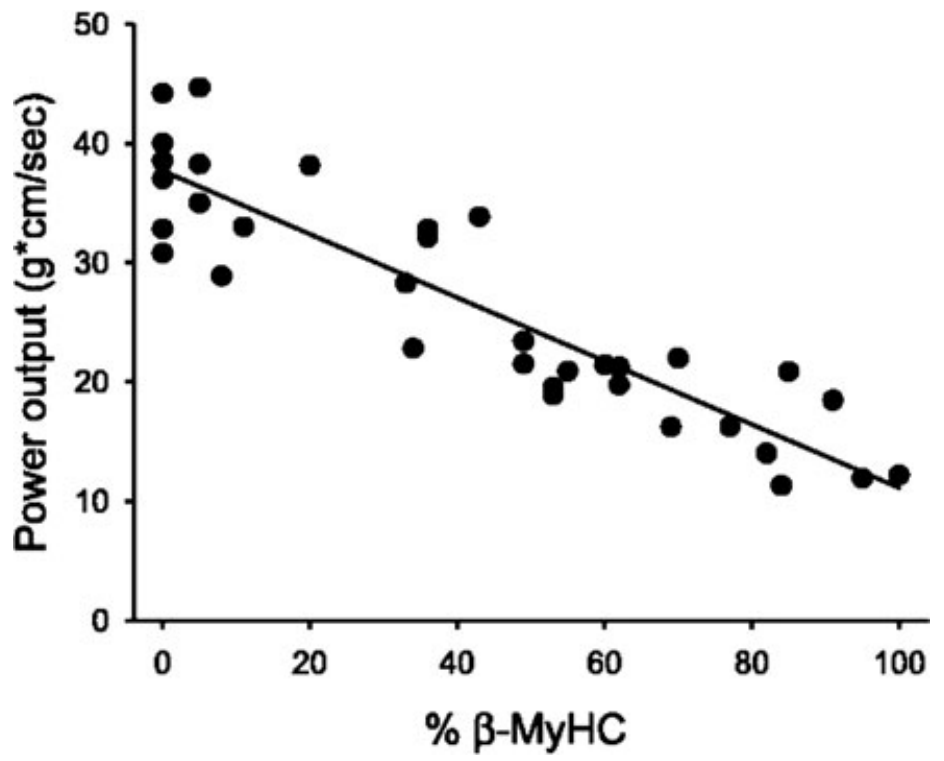

FIGURE 2. Power output of the whole working heart is reduced as the relative expression of $\beta-M y H C$ increases. (From Korte et al. ${ }^{26}$ Reproduced by permission).

been shown to develop upregulation of levels of $\alpha$ MyHC. ${ }^{31-33}$ These data suggest that levels of $\alpha-\mathrm{MyHC}$ serve at the least as a surrogate marker for cardiac function in heart failure, but this hypothesis has been difficult to test directly. In animal models, even a small increase in expression of $\alpha-\mathrm{MyHC}$ results in an increase in power output by the heart. ${ }^{34}$ In a rabbit model of induced cardiomyopathy, overexpression of an $\alpha-\mathrm{MyHC}$ transgene was found to be protective. ${ }^{35} \mathrm{Also}$, in another transgenic model, expression of $\beta-\mathrm{MyHC}$, with concomitant downregulation of $\alpha-\mathrm{MyHC}$, was found to have a detrimental effect on mice that were placed under cardiovascular stress. ${ }^{36}$ Taken together, the evidence suggests that acute upregulation of $\alpha-\mathrm{MyHC}$ expression may lead to improved cardiac function, while increases in $\beta$-MyHC expression may be detrimental to cardiac performance. Study of the direct effects of increased fast $\alpha-\mathrm{MyHC}$ motor expression will require the use of acute gene transfer of MYH6 (the gene that encodes $\alpha-\mathrm{MyHC}$ ) to failing cardiac myocytes in vitro and to failing hearts in vivo.

Strategies that have been employed to investigate the functional significance of myosin isoform switching have inherent limitations. The most commonly used model has been the rodent hypothyroid animal model. Thyroid hormone is a potent inducer of $\alpha$ MyHC expression and in the absence of thyroid hormone, by thyroidectomy or propyl-thio-uracil (PTU) treatment, there is a well documented reduction of $\alpha$-MyHC expression accompanied by an increase of
$\beta$-MyHC expression. ${ }^{14,37}$ Studies using hypothyroid rodents have provided evidence that increased $\beta$ $\mathrm{MyHC}$ expression depresses contractile function of single cardiac myocytes ${ }^{24,25,34,38}$ and in the whole heart. ${ }^{23,39}$ However, a key limitation of the hypothyroid model is that in concert with alterations in the MyHC isoform profile, the expression of key calcium handling proteins, including the SR $\mathrm{Ca}^{2+}$-ATPase (SERCA2a), are also affected. ${ }^{40,41}$ Thus, interpretation of functional data is confounded because SERCA2a activity itself is well known to markedly affect cardiac performance. ${ }^{42-44}$ To address this confounding issue, which is attributed to altered calcium handling, the permeabilized (skinned) myocyte preparation, a preparation that eliminates any contribution of calcium-handling proteins (like SERCA2a) to myocyte functional studies, has been enlisted. ${ }^{24,25}$ These studies have demonstrated that mechanical properties of single myocytes (e.g., power output and unloaded shortening velocity) are attenuated by increased relative expression of $\beta$-MyHC. While these studies provided important information about the contribution of myosin isoforms to myofilament function, they do not address the role of myosin isoform switching in the more physiologically relevant setting of the electrically stimulated intact cardiac muscle cell where excitationcontraction coupling mechanisms are intact and fully functional.

Chronic transgenic animal models have also been used to manipulate $\mathrm{MyHC}$ isoform expression in the 
mammalian heart in vivo. ${ }^{35,36,45}$ Under baseline conditions, the near full replacement of $\alpha-\mathrm{MyHC}$ with $\beta$ $\mathrm{MyHC}$ in the hearts of transgenic mice had no detected effects on echocardiography-derived shortening fraction, an in vivo measure of systolic function. ${ }^{36}$ Isolated permeabilized muscle preparations from these mice, however, did show adverse effects on contractile performance at baseline. It is possible that chronic forced genetic transition from $\alpha-\mathrm{MyHC}$ to $\beta$-MyHC throughout the development of these animals may have caused other adaptations in the mouse heart to compensate for effects of $\beta$-MyHC expression in vivo. This is conceivable, considering that these transgenic mice would have sustained nearly 150 million contractile cycles in vivo prior to cardiac performance assessment in adult mice. In transgenic animal models, therefore, it may be difficult to distinguish primary effects caused by forced $\beta$-MyHC expression from secondary compensatory changes that may occur throughout the development and lifetime of a transgenic animal. ${ }^{46}$ The utilization and development of acute gene transfer of myosin molecular motors circumvents many of the complication(s) posed by secondary compensatory alterations of protein expression that may have altered or masked a direct effect of $\mathrm{MyHC}$ isoform switching on cardiac performance.

\section{Myosin Isoform Switching: Acute Gene Transfer}

We recently used acute genetic engineering to study the direct effect of increased relative $\beta-\mathrm{MyHC}$ expression on single membrane intact cardiac myocyte function. ${ }^{47}$ Experimentally we designed and generated a recombinant adenovirus, AdMYH7, to express the full-length human MYH7 (full-length $\beta$-MyHC molecule) gene in $\alpha$-MyHC-dominant rat myocytes in vitro. The efficiency of gene transfer was $\sim 100 \%$ and stoichiometric replacement of the endogenous $\alpha-$ MyHC motor with the full length human $\beta$-MyHC motor was shown by Western blot analysis. Proper sarcomeric incorporation of virally directed $\beta$-MyHC expression was detected in the A-band of the sarcomere by using indirect immunofluorescence and high-resolution confocal imaging. Functional effects of $\beta$-MyHC gene transfer were determined in electrically paced myocytes by measuring changes in sarcomere spacing and intracellular calcium transients using fura-2. $\beta$-MyHC gene transfer attenuated myocyte contractility just 1 day after gene transfer (FIG. 3), when $\beta$-MyHC accounted for just $18 \%$ of the total myosin (FIG. 3). This functional effect was transgene dose-dependent as the effect on contraction amplitude was greater on day 2 , when $\beta-\mathrm{MyHC}$ accounted for $\sim 40 \%$ of the total myosin. The amplitude of the electrically stimulated intracellular calcium transient, however, was not affected by $\beta$-MyHC expression. This study demonstrated for the first time that increased expression of the heart's slow $\beta$-MyHC molecular motor can have calcium-independent negative inotropic effects on single cardiac myocyte contractility. Thus, in addition to being a common molecular marker for heart failure, increased $\beta$-MyHC expression can directly contribute to cardiac dysfunction. Acute gene transfer of molecular motors in cardiac muscle offers a novel means of calcium independent inotropy.

\section{Cardiac Myosin and Inherited Cardiomyopathies}

Inherited cardiomyopathy can be caused by mutations of sarcomere proteins. The one most commonly affected sarcomeric protein in familial cardiomyopathy is $\beta$-MyHC. Mutations of the MYH7 gene, the gene that encodes $\beta$-MyHC, have been associated with both HCM and DCM. Although the human heart expresses predominantly $\beta-\mathrm{MyHC}$, mutations of MYH6, the gene that encodes $\alpha-\mathrm{MyHC}$, have recently been reported to also cause either HCM or DGM. ${ }^{8}$ The clinical importance of MYH7 mutations is underscored by the finding that MYH7 mutations have also been linked to skeletal muscle myopathies, including Laing distal myopathy, ${ }^{9}$ myosin storage myopathy, ${ }^{48}$ and hyaline body myopathy. ${ }^{49}$ In fact, a report from the American Heart Association has suggested that for cardiovascular genetic counseling, genetic screening should start with examination of the MYH7 gene. $^{50}$

The first HCM-causing myosin mutation was discovered in the motor domain of the $\beta$-MyHC molecule. ${ }^{6}$ This missense mutation results in an arginine-to-glutamine substitution at position 403 (R403Q), which is located in the actin-binding interface of the N-terminal globular head. ${ }^{51}$ This mutation results in the loss of a positively charged amino acid residue in the actin-binding site. All affected members of this large kindred were heterozygous for the R403Q mutation, and unaffected family members did not carry this mutation. The R403Q mutation is associated with early onset of symptoms and a high mortality rate, with an average life span of 30-35 years. ${ }^{52,53}$ Since the discovery of this myosin mutation, much effort has been devoted to determining how it affects 
$\mathbf{A}$
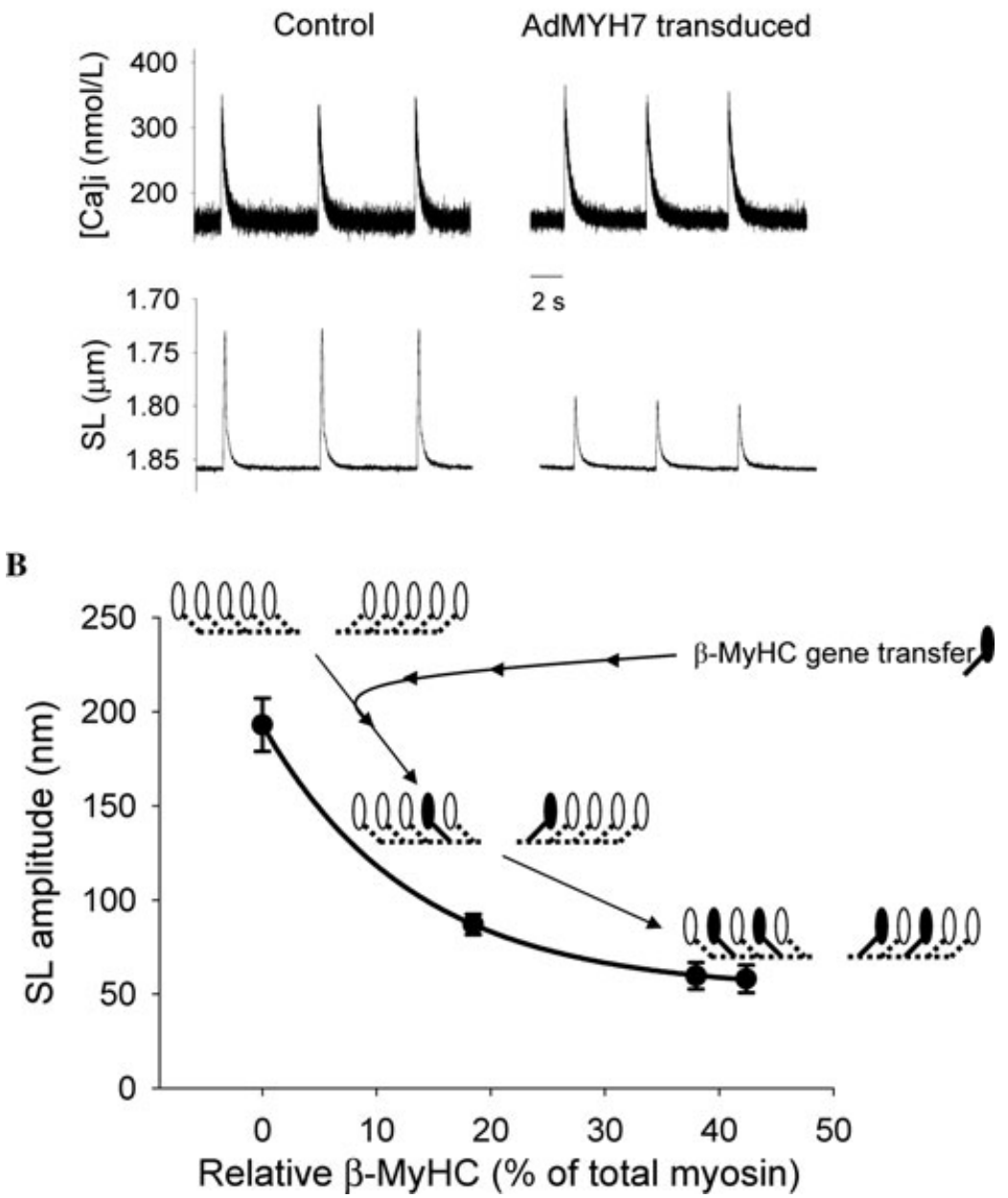

FIGURE 3. (A) Gene transfer of the slow $\beta-M y H C$ motor directly attenuates cardiac myocyte contractility independent of the intracellular calcium transient. ${ }^{47}$ (B) Increasing relative $\beta-M y H C$ expression by acute gene transfer attenuates contractility in a dose-dependent manner. $\mathrm{SL}=$ sarcomere length.

myosin motor function and triggers cardiomyopathy, but the issue remains unresolved as some studies have indicated that motor function is impaired, ${ }^{54-57}$ while others suggest a gain of function for the myosin motor. ${ }^{58-61}$ The links between mutated myosin (R403Q) function and cardiac hypertrophy are unclear, though some studies have suggested a mechanism involving altered calcium homeostasis. ${ }^{62-64}$ The role of calcium in the pathogenesis initiated by R403Q myosin is unclear as it has been reported that transgenic animals expressing R403Q have elevated calcium levels, ${ }^{63}$ while other reports suggest depressed calcium levels in heart cells from these animals. ${ }^{62,64}$ One limitation of these elegant studies is that the R403Q mutation was studied in the context of the mouse $\alpha$-MyHC molecule
(MYH6 gene), while the disease-causing mutation in humans occurs in the context of the MYH7 gene ( $\beta$ MyHC molecule). As outlined above, $\alpha$ - and $\beta-\mathrm{MyHC}$ are structurally and functionally distinct, thus making it conceivable that a point mutation in one molecule may not affect the other in the same way. A transgenic rabbit, whose myosin isoform expression profile is similar to that of humans, has been developed which expresses the R403Q mutant myosin in the context of the human MYH7 gene. ${ }^{65}$ This model recapitulates much of the phenotype observed in humans (e.g., fibrosis, cardiac hypertrophy, whole-organ dysfunction), but the acute effect of this mutant myosin on cardiac performance (single-cell contractility and calcium homeostasis) has not been studied in this model. 


\section{Acute Gene Transfer of HCM Mutant Myosin}

Two studies from different laboratories have used acute gene transfer technology to study the effect of the HCM disease-causing R403Q mutant myosin on cardiac tissue. In 1995, Marian et al. ${ }^{66}$ used a replicationdeficient recombinant adenoviral construct to deliver wild-type and mutated (R403Q) human $\beta$-MyHC to feline cardiac myocyte in vitro. The advantage of this approach is that the feline species is known to develop HCM with a phenotype identical to that in humans and that the investigators generated this mutant myosin in the context of the bona fide disease-causing gene (MYH7). The efficiency of in vitro gene transfer was reportedly high $(>95 \%)$, and the mutant myosin caused sarcomere disruption 120 hours after gene transfer. While this study indicated that disruption of sarcomere assembly and myofibrillar organization due to mutant $\beta-\mathrm{MyHC}$ is a primary defect in HCM, it did not assess the acute functional effect of this mutant myosin on cardiac performance (i.e., single-cell contractility or intracellular calcium homeostasis.).

In 2003, Wang et al. ${ }^{67}$ investigated the functional impact of mutant myosin on cardiac myofibril organization and myosin motor activity. Using embryonic chicken myosin fused to a GFP reporter, Wang et al. found that expression of the R403Q mutant myosin accelerated actin filament sliding, but decreased myosin's affinity for actin in an in vitro motility assay. This is consistent with previous reports that suggest augmentation of myosin function due to the R403Q mutation. ${ }^{58-61}$ Insight from this study, however, is limited since the myosin used was tagged with GFP, a fluorescent molecule that can have direct effects on actomyosin interactions. ${ }^{68}$ Further, this study performed acute gene transfer of mutant myosin in chicken embryonic cardiac myocytes and in a mouse myogenic cell line (C2C12) and not in adult mammalian cardiac myocytes. The direct effects of mutant myosin on adult cardiac myocyte contractility and calcium homeostasis using acute gene transfer technology are largely unexplored. Determination of the acute effects of mutant myosins on adult myocyte function will provide important insight into molecular triggers that initiate and contribute to cardiac disease.

\section{Summary}

Cardiac molecular motors are important regulators of cardiac performance. Cardiac muscle contraction is initiated by calcium binding to and activating the thin filament, thus promoting molecular motor (myosin) activity and force generation. While the amount of force generation can be graded with calcium, myocyte contractility can also be altered by the motor protein isoform expression profile. Experimentally, acute gene transfer of the slow $\beta$-MyHC molecular motor provides a means of calcium-independent negative inotropy in single cardiac myocytes. Acute gene transfer of the fast $\alpha-\mathrm{MyHC}$ molecular motor may provide a means of calcium-independent positive inotropy, though this remains to be tested in the laboratory. While acute gene transfer of molecular motors is in its infancy, future research aimed at using myosin gene transfer as a therapeutic agent for the failing heart will require development of safe and effective vectors for delivery of the myosin gene $(\sim 6 \mathrm{~kb})$ to the heart in vivo. The study of acute functional effects of disease-causing myosin mutations that trigger cardiomyopathy will provide mechanistic insight into the molecular mechanisms that initiate heart disease.

\section{Conflict of Interest}

The authors declare no conflicts of interest.

\section{References}

1. Hinken, A.C. \& R.J. Solaro. 2007. A dominant role of cardiac molecular motors in the intrinsic regulation of ventricular ejection and relaxation. Physiology 22: 73 80.

2. MitkA, M. 2006. Do lackluster trial findings mean new avenues are needed for heart research? JAMA 295: 611 612.

3. Lowes, B.D., W. Minobe, W.T. Abraham, et al. 1997. Changes in gene expression in the intact human heart: downregulation of alpha-myosin heavy chain in hypertrophied, failing ventricular myocardium. J. Clin. Invest. 100: 2315-2324.

4. Miyata, S., W. Minobe, M.R. Bristow \& L.A. Leinwand. 2000. Myosin heavy chain isoform expression in the failing and nonfailing human heart. Circ. Res. 86: 386-390.

5. NakaO, K., W. Minobe, R. Roden, et al. 1997. Myosin heavy chain gene expression in human heart failure. J. Clin. Invest. 100: 2362-2370.

6. Geisterfer-Lowrance, A.A., S. Kass, G. Tanigawa, et al. 1990. A molecular basis for familial hypertrophic cardiomyopathy: a beta cardiac myosin heavy chain gene missense mutation. Cell 62: 999-1006.

7. Fatkin, D. \& R.M. Graham. 2002. Molecular mechanisms of inherited cardiomyopathies. Physiol. Rev. 82: 945-980.

8. Garniel, E., M.R.G. TAYlor, G. Sinagra, et al. 2005. $\alpha-$ Myosin heavy chain: a sarcomeric gene associated with dilated and hypertrophic phenotypes of cardiomyopathy. Circulation 112: $54-59$.

9. Meredith, C., R. Herrmann, C. Parry, et al. 2004. Mutations in the slow skeletal muscle fiber myosin heavy chain 
gene (MYH7) cause Laing early-onset distal myopathy (MPD1). Am. J. Hum. Genet. 75: 703-708.

10. Rayment, I., W.R. Rypniewski, K. Schmidt-Base, et al. 1993. Three-dimensional structure of myosin subfragment-1: a molecular motor. Science 261: 50-58.

11. Нон, J.F.Y., G.P.S. YeOH, M.A.W. Thomas, et al. 1979. Structural differences in the heavy chains of rat ventricular myosin isoenzymes. FEBS Lett. 97: 330-334.

12. Pope, B., J.F.Y. HoH \& A. Weeds. 1980. The ATPase activities of rat cardiac myosin isoenzymes. FEBS Lett. 118: 205-208.

13. Saez, L.J., K.M. Gianola, E.M. McNally, et al. 1987. Human cardiac myosin heavy chain genes and their linkage in the genome. Nucleic Acids Res. 15: 5443-5459.

14. Haddad, F., P.W. Bodell, A.X. Qin, et al. 2003. Role of antisense RNA in coordinating cardiac myosin heavy chain gene switching. J. Biol. Chem. 278: 37132-37138.

15. Lompre, A.M., B. NADAL-Ginard \& V. MahdaVi. 1984. Expression of cardiac alpha and beta-myosin heavy chain genes is developmentally and hormonally regulated. J. Biol. Chem. 259: 6437-6446.

16. Harris, D.E., S.S. WOrk, R.K. Wright, et al. 1994. Smooth, cardiac, and skeletal muscle myosin force and motion generation assessed by cross-bridge mechanical interactions in vitro. J. Muscle Res. Cell Motil. 15: 11-19.

17. Van Buren, P., D.E. Harris, N.R. Alpert. 1995. Cardiac V1 and V3 myosins differ in their hydrolytic and mechanical activities in vitro. Circ. Res. 77: 439-444.

18. Malmovist, U.P., A. Aronshtam \& S. Lowey. 2004. Cardiac myosin isoforms from different species have unique enzymatic and mechanical properties. Biochemistry 43: 15058-15065.

19. Palmiter, K.A., M.J. Tyska, D.E. Dupuis, et al. 1999. Kinetic differences at the single molecule level account for the functional diversity of rabbit cardiac myosin isoforms. J. Physiol. (Lond.) 519: 669-678.

20. Hasenfuss, G., L.A. Mulieri, E.M. Blanchard, et al. 1991. Energetics of isometric force development in control and volume- overload human myocardium: comparison with animal species. Circ. Res. 68: $836-846$.

21. Holubarsch, C., R.P. Goulette, R.Z. Litten, et al. 1985. The economy of isometric force development, myosin isoenzyme pattern and myofibrillar ATPase activity in normal and hypothyroid rat myocardium. Circ. Res. 56: 78-86.

22. Rundell, V.L.M., V. Manaves, A.F. Martin, et al. 2005. Impact of $\beta$-myosin heavy chain isoform expression on cross-bridge cycling kinetics. Am. J. Physiol. Heart Circ. Physiol. 288: H896-H903.

23. TANG, Y.D., J.A. KuZman, S. SAID, et al. 2005. Low thyroid function leads to cardiac atrophy with chamber dilatation, impaired myocardial blood flow, loss of arterioles, and severe systolic dysfunction. Circulation 112: $3122-$ 3130.

24. Herron, T.J., F.S. Korte \& K.S. MaDonald. 2001. Loaded shortening and power output in cardiac myocytes are dependent on myosin heavy chain isoform expression. Am. J. Physiol. Heart Circ. Physiol. 281: H1217-H1222.

25. Metzger, J.M., P.A. Wahr, D.E. Michele, et al. 1999. Effects of myosin heavy chain isoform switching on $\mathrm{Ca}^{2+}$ - activated tension development in single adult cardiac myocytes. Circ. Res. 84: 1310-1317.

26. Korte, F.S., T.J. Herron, M.J. Rovetto, et al. 2005. Power output is linearly related to MyHC content in rat skinned myocytes and isolated working hearts. Am. J. Physiol. Heart. Circ. Physiol. 289: H801-H812.

27. Rundell, V.L.M., D.L. GEenen, P.M. Buttrick, et al. 2004. Depressed cardiac tension cost in experimental diabetes is due to altered myosin heavy chain isoform expression. Am. J. Physiol. Heart Circ. Physiol. 287: H408-H413.

28. Mercadier, J.J., A.M. Lompre, G. Wisnewsky, et al. 1981. Myosin isoenzymic changes in several models of rat cardiac hypertrophy. Circ. Res. 49: 525-532.

29. Fitzsimons, D.P., J.R. Patel \& R.L. Moss. 1999. Agingdependent depression in the kinetics of force development in rat skinned myocardium. Am. J. Physiol. Heart Circ. Physiol. 276: H1511-H1519.

30. Masaki, H., T. Imaizumi, S. Ando, et al. 1993. Production of chronic congestive heart failure by rapid ventricular pacing in the rabbit. Cardiovasc. Res. 27: 828-831.

31. Wang, J., X. Guo \& N.S. Dhalla. 2004. Modification of myosin protein and gene expression in failing hearts due to myocardial infarction by enalapril or losartan. Biochim. Biophys. Acta 1690: 177-184.

32. Lowes, B.D., E.M. Gilbert, W.T. Abraham, et al. 2002. Myocardial gene expression in dilated cardiomyopathy treated with beta-blocking agents. N. Engl. J. Med. 346: 1357-1365.

33. Rastogi, S., S. Mishra, R. Gupta, et al. 2005. Reversal of maladaptive gene program in left ventricular myocardium of dogs with heart failure following long-term therapy with the Acorn cardiac support device. Heart Failure Rev. 10: 157-163.

34. Herron, T.J. \& K.S. MaDonald. 2002. Small amounts of $\alpha$-myosin heavy chain isoform expression significantly increase power output of rat cardiac myocyte fragments. Circ. Res. 90: 1150-1152.

35. James, J., L. Martin, M. Krenz, et al. 2005. Forced expression of $\alpha$-myosin heavy chain in the rabbit ventricle results in cardioprotection under cardiomyopathic conditions. Circulation 111: 2339-2346.

36. Krenz, M. \& J. Robisns. 2004. Impact of beta-myosin heavy chain expression on cardiac function during stress. J. Am. Coll. Cardiol. 44: 2390-2397.

37. Izumo, S., B. Nadal-Ginard \& V. MahdaVI. 1986. All members of the MHC multigene family respond to thyroid hormone in a highly tissue-specific manner. Science 231: $597-600$.

38. Fitzsimons, D.P., J.R. Patel \& R.L. Moss. 1998. Role of myosin heavy chain composition in kinetics of force development and relaxation in rat myocardium. J. Physiol. (Lond.) 513: 171-183.

39. Korte, F.S., T.J. Herron, M.J. Rovetto, et al. 2005. Power output is linearly related to myosin heavy chain content in rat skinned myocytes and isolated working hearts. Am. J. Physiol. Heart Circ. Physiol. 289: H801-H812.

40. Sayen, M.R., D.K. Rohrer \& W.H. Dillmann. 1992. Thyroid hormone response of slow and fast sarcoplasmic reticulum $\mathrm{Ca}^{2+}$ ATPase mRNA in striated muscle. Mol. Cell. Endocrinol. 87: 87-93. 
41. Carr, A.N, \& E.G. Kranias. 2002. Thyroid hormone regulation of calcium cycling proteins. Thyroid 12: 453-457.

42. Arai, M., N.R. Alpert, D.H. MacLennan, et al. 1993. Alterations in sarcoplasmic reticulum gene expression in human heart failure: a possible mechanism for alterations in systolic and diastolic properties of the failing myocardium. Circ. Res. 72: 463-469.

43. Hasenfuss, G., H. Reinecke, R. Studer, et al. 1994. Relation between myocardial function and expression of sarcoplasmic reticulum $\mathrm{Ca}(2+)$-ATPase in failing and nonfailing human myocardium. Circ. Res. 75: 434- 442.

44. Teucher, N., J. Prestle, T. Seidler, et al. 2004. Excessive sarcoplasmic/endoplasmic reticulum $\mathrm{Ca}^{2+}$-ATPase expression causes increased sarcoplasmic reticulum $\mathrm{Ca}^{2+}$ uptake but decreases myocyte shortening. Circulation 110: 3553-3559.

45. TARdiff, J.C., T.E. HewetT, S.M. Factor, et al. 2000. Expression of the beta (slow)-isoform of MHC in the adult mouse heart causes dominant-negative functional effects. Am. J. Physiol. Heart Circ. Physiol. 278: H412-H419.

46. Michele, D.E. \& J.M. Metzger,2000. Contractile dysfunction in hypertrophic cardiomyopathy: elucidating primary defects of mutant contractile proteins by gene transfer. Trends Cardiovasc. Med. 10: 177-182.

47. Herron, T.J., R. Van Den Boom, E. Fomicheva, et al. 2007. Calcium-independent negative inotropy by $\beta$-myosin heavy chain gene transfer in cardiac myocytes. Circ. Res. 100: 1182-1190.

48. Tajsharghi, H., L.E. Thornell, C. LindberG, et al. 2004. Myosin storage myopathy associated with a heterozygous missense mutation in MYH7. Ann. Neurol. 54: 494-500.

49. Bohlega, S., S.N. Bu-Amero, S.M. WAKIL, et al. 2004. Mutation of the slow myosin heavy chain rod domain underlies hyaline body myopathy. Neurology 62: 15181521.

50. Richard, P., P. Charron, L. Carrier, et al. 2003. Hypertrophic cardiomyopathy: distribution of disease genes, spectrum of mutations, and implications for a molecular diagnosis strategy. Circulation 107: 2227-2232.

51. Rayment, I., H.M. Holden, J.R. Sellers, et al. 1995. Structural interpretation of the mutations in the $\beta$-cardiac myosin that have been implicated in familial hypertrophic cardiomyopathy. Proc. Natl. Acad. Sci. USA 92: 3864 3868.

52. Fananapazir, L., M.C. Dalakas, F. Gyran, et al. 1993. Missense mutations in the $\beta$-myosin heavy-chain gene cause central core disease in hypertrophic cardiomyopathy. Proc. Natl. Acad. Sci. USA 90: 3993-3997.

53. Watkins, H., A. Rosenzweig, D.S. Hwanng, et al. 1992. Characteristics and prognostic implications of myosin missense mutations in familial hypertrophic cardiomyopathy. N. Engl. J. Med. 326: 1108-1114.

54. Blanchard, E., C. Seidman, J.G. Seidman, et al. 1999. Altered crossbridge kinetics in the $\alpha$ MHC403/+ mouse model of familial hypertrophic cardiomyopathy. Circ. Res. 84: $475-483$.

55. Cuda, G., L. Fananapazir, W.S. Zhu, et al. 1993. Skeletal muscle expression and abnormal of beta-myosin in hypertrophic cardiomyopathy. J. Clin. Invest. 91: 28612865.

56. Cuda, G., L. Fananapazir, N.D. Epstein, et al. 1997. The in vitro motility activity of beta-cardiac myosin depends on the nature of the beta myosin heavy chain gene mutation in hypertrophic cardiomyopathy. J. Muscle Res. Cell Motil. 18: $275-283$.

57. Sweeney, H.L., A.J. Straceski, L.A. Leinwand, et al. 1994. Heterologous expression of a cardiomyopathic myosin that is defective in its actin interaction. J. Biol. Chem. 269: 1603-1605.

58. Palmer, B.M., D.E. Fishbaugher, J.P. Schmitt, et al. 2004. Differential cross-bridge kinetics of FHC myosin mutations R403Q and R453C in heterozygous mouse myocardium. Am. J. Physiol. Heart Circ. Physiol. 287: H91H99.

59. Tyska, M.J., E. Hayes, M. Giewat, et al. 2000. Single-molecule mechanics of R403Q cardiac myosin isolated from the mouse model of familial hypertrophic cardiomyopathy. Circ. Res. 86: 737744.

60. Yamashita, H., M.J. Tyska, D.M. Warshaw, et al. 2000. Functional consequences of mutations in the smooth muscle myosin heavy chain at sites implicated in familial hypertrophic cardiomyopathy. J. Biol. Chem. 275: 2804528052.

61. Debold, E.P., J.P. Schmitt, J.R. MoORe, et al. 2007. Hypertrophic and dilated cardiomyopathy mutations differentially affect the molecular force generation of mouse $\alpha$-cardiac myosin in the laser trap assay. Am. J. Physiol. Heart Circ. Physiol. 00128.

62. Fatkin, D., B.K. McConnell, J.O. Mudd, et al. 2000. An abnormal $\mathrm{Ca}^{2+}$ response in mutant sarcomere proteinmediated familial hypertrophic cardiomyopathy. J. Clin. Invest. 106: 1351-1359.

63. GaO, W.D., N.G. Perez, C.E. Seidman, et al. 1999. Altered cardiac excitation-contraction coupling in mutant mice with familial hypertrophic cardiomyopathy. J. Clin. Invest. 103: $661-666$.

64. Semsarian, C., I. Ahmad, M. Giewat, et al. 2002. The L-type calcium channel inhibitor diltiazem prevents cardiomyopathy in a mouse model.J. Clin. Invest. 109: 10131020.

65. Marian, A.J., Y. WU, D.S. Lim, et al. 1999. A transgenic rabbit model for human hypertrophic cardiomyopathy. J. Clin. Invest. 104: 1683-1692.

66. Marian, A.J., Q.T. YU, D.L. Mann, et al. 1995. Expression of a mutation causing hypertrophic cardiomyopathy disrupts sarcomere assembly in adult feline cardiac myocytes. Circ. Res. 77: 98-106.

67. Wang, Q., G.L. Moncman \& D.A. Winkelmann. 2003. Mutations in the motor domain modulate myosin activity and myofibril organization. J. Cell Sci. 116: $4227-$ 4238 .

68. Agbulut, O., A. Huet, N. Niederlander, et al. 2007. Green fluorescent protein impairs actin-myosin interactions by binding to the actin-binding site of myosin. J. Biol. Chem. 282: 10465-10471. 